\title{
Familial Aggregation of Cranial Tremor in Familial Essential Tremor
}

\author{
Elan D. Louis ${ }^{a-d}$ Nora Hernandez ${ }^{a}$ Lorraine N. Clark ${ }^{c, e}$ Ruth Ottman ${ }^{a}, b, d, f$ \\ ${ }^{a}$ G.H. Sergievsky Center, ${ }^{b}$ Department of Neurology, and ${ }^{\mathrm{C}}$ Taub Institute for Research on Alzheimer's Disease and \\ the Aging Brain, College of Physicians and Surgeons, Columbia University, ${ }^{d}$ Department of Epidemiology, Mailman \\ School of Public Health, Columbia University, ${ }^{e}$ Department of Pathology and Cell Biology, Columbia University

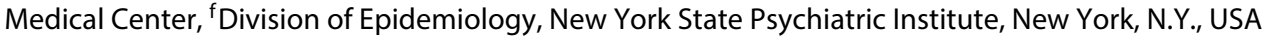

\author{
Key Words \\ Essential tremor - Genetics · Familial tremor · Clinical \\ correlates $\cdot$ Cranial tremor $\cdot$ Head tremor
}

\begin{abstract}
Background: Essential tremor (ET) is often familial and phenotypic features may be shared within families. Cranial (neck, voice, and jaw) tremor is an important feature of ET. We examined whether cranial tremor aggregates in ET families, after controlling for other factors (age, tremor severity, and duration). Methods: Among ET probands and relatives enrolled in a genetic study at Columbia University (95 subjects in 28 families), we assessed the degree to which occurrence of cranial tremor in the proband predicted occurrence of cranial tremor in affected relatives. Results: Forty-five (47.4\%) subjects had cranial tremor on neurological examination (probands $66.7 \%$, relatives $39.7 \%$ ). Among 28 families, 23 (82.1\%) contained individuals with and individuals without cranial tremor, indicating a high degree of within-family heterogeneity. In comparison to subjects without cranial tremor, those with cranial tremor had higher total tremor scores ( $p$ $<0.001)$, were older ( $p=0.003)$, and had tremor of longer duration $(p=0.01)$. In logistic regression models, the odds of cranial tremor in a relative were not related to occurrence of
\end{abstract}

cranial tremor in the proband ( $p>0.24)$. Conclusions: Cranial tremor did not aggregate in families with ET; the major predictor of this disease feature was tremor severity rather than presence of cranial tremor in another family member.

Copyright $\odot 2013$ S. Karger AG, Basel

\section{Introduction}

Cranial tremor, including neck, voice, and jaw tremor, is an important feature of essential tremor (ET). Following upper limb tremor, it stands out as the most common site of tremor in this disease [1-3]. It also has several important clinical correlates; for example, it has been found to be associated with greater gait and balance difficulty [4-6] and may be more resistant to treatment than other tremor types [7-9]. Head and jaw tremor are also visually quite apparent and of particular cosmetic concern for patients.

ET is often familial [10]. In the ongoing search for susceptibility genotypes [11], the study of its manifestations within families is currently an area of considerable research focus.

Until recently, the familial aggregation of the phenotypic features of ET has received little attention. We re-

\section{KARGER}

E-Mail karger@karger.com

www.karger.com/ned
(C) 2013 S. Karger AG, Basel

0251-5350/13/0411-0048\$38.00/0
Dr. Elan Louis

Unit 198, Neurological Institute

710 West 168th Street

New York, NY 10032 (USA)

E-Mail EDL2@columbia.edu 
cently found that age of onset and rate of progression aggregate in ET families $[12,13]$. Despite the high prevalence of cranial tremor, there are no readily available data on the concordance for such tremor within ET patients and their families. This is an issue that has research as well as clinical ramifications. ET patients whose relatives (e.g. parents) have/had head tremor often ask whether they, too, are likely to develop a similar feature. Data to guide clinicians on how to answer this question are lacking.

In the current investigation, our goal was to study the familial aggregation of cranial tremor in families containing multiple individuals with ET, enrolled in a genetic study of ET at Columbia University Medical Center (CUMC) [14]. We also considered whether several other factors (age, tremor severity, and duration) predicted occurrence of cranial tremor. Our hypothesis was that the presence of cranial tremor in one or more family members would strongly predict the presence of such tremor in their relatives, after controlling for other factors. Given the higher prevalence of cranial tremor among women with ET [15], we also expected that female gender would predict presence of cranial tremor.

\section{Methods}

\section{Ascertainment of Probands}

ET cases (probands) and their reportedly affected first- and second-degree relatives were enrolled in a genetic study of ET, the Family Study of Essential Tremor (FASET) at CUMC [14]. The study was advertised on two ET society websites. The three initial inclusion criteria for probands were: (1) a diagnosis of ET had been assigned by a doctor; (2) age of tremor onset was $\leq 40$ years (this was later changed to $\leq 50$ years to be more inclusive), and (3) $\geq 2$ living relatives in the United States with ET also diagnosed by a doctor and who were not reported to have dystonia or Parkinson's disease (PD). The exclusion criterion for probands was a prior diagnosis of dystonia or $\mathrm{PD}$. The rationale for enrolling probands whose tremor onset was $\leq 40$ years was to increase the likelihood of enrolling probands with underlying susceptibility genotypes of ET. ET patients with younger age of tremor onset often have the familial form of the disease. Indeed, we previously reported that a large proportion of the young-onset cases had the familial form of ET [16].

Potential ET probands contacted the FASET study coordinator. Prior to final selection for enrollment, a set of four Archimedean spirals (two right, two left) was submitted by probands and rated by a senior neurologist specializing in movement disorders (E.D.L.). Probands were included if one or more of the spirals had a Washington Heights Inwood Genetic Study of Essential Tremor rating of 2 (moderate tremor) or higher [17].

\section{Ascertainment of Relatives}

Based upon a telephone interview with the proband, relatives with ET were identified [14]. With the proband's permission, these relatives were then contacted by telephone and were pre-enrolled if they reported the presence of tremor in the absence of a prior diagnosis of dystonia or PD. Prior to final selection for enrollment, relatives submitted four Archimedean spirals. These spirals were rated (E.D.L.), and relatives were included if one or more of the spirals had a rating $\geq 2$ [17].

\section{Evaluation}

An in-person evaluation was then conducted in the enrollees' homes; this included a series of questionnaires and a videotaped neurological examination [14]. Age of tremor onset was by selfreport and was the age at which the individual first noted tremor. Prior studies have indicated that it is reliably reported by ET patients [18]. Tremor duration was the difference between current age and age of tremor onset. The videotaped neurological examination included a detailed assessment of postural, kinetic, intention, and rest tremors in the limbs, as well as dystonia and other movement disorders [19]. Voice tremor was assessed during sustained phonation, conversational speech, and while reading a prepared passage. Neck (i.e. head) tremor was assessed while seated comfortably and facing the camera. Jaw tremor was assessed while the mouth was stationary (closed), while the patient was asked to hold their mouth slightly open, during sustained phonation, and during speech [3]. The neurologist (E.D.L.) reviewed all videotaped examinations and rated the severity of postural and kinetic arm tremors (0-3), resulting in a total tremor score [range 0-36 (maximum)] [19]. The study was approved by the CUMC Institutional Review Board and all participants gave written informed consent.

\section{Diagnoses}

All ET diagnoses were reconfirmed based on review of questionnaires and videotaped neurological examinations [14]. Diagnoses of ET were assigned based on published diagnostic criteria (moderate or greater amplitude kinetic tremor during three or more activities or a head tremor in the absence of PD or another known cause) [17, 20].

\section{Final Sample}

There were 145 enrollees ( 38 probands and 107 relatives). For the current analyses, we excluded 47 enrollees for the following reasons: 26 families did not contain $\geq 1$ relatives with ET, 22 had dystonia (mainly torticollis), and 2 had incomplete data. Hence, the final sample consisted of 27 probands and 68 relatives (95 ET total). These 95 included 1 family (family 23) in which there were 4 relatives, but the proband had dystonia, so only the data on the 4 relatives are presented here. The final sample of 95 (table 1) was similar to the initial sample of 145 in terms of age ( $56.3 \pm 18.5$ vs. $58.5 \pm 18.0$ years, $\mathrm{t}=0.92, \mathrm{p}=0.36)$, gender $[49(51.6 \%)$ vs. 86 (59.3\%) female, $\left.\chi^{2}=1.39, \mathrm{p}=0.24\right]$, tremor duration $(28.3 \pm 18.9$ vs. $29.6 \pm 20.0$ years, $\mathrm{t}=0.50, \mathrm{p}=0.62)$, total tremor score $(18.5 \pm$ 6.3 vs. $17.5 \pm 7.0, \mathrm{t}=1.13, \mathrm{p}=0.26)$, and proportion with cranial tremor [ $45(47.4 \%)$ vs. $\left.58(40.0 \%), \chi^{2}=1.27, \mathrm{p}=0.26\right]$.

\section{Statistical Analyses}

Analyses were performed in SPSS (version 19.0). Subject characteristics were compared using Student's t tests and $\chi^{2}$ tests. Correlations between continuous variables were assessed with Pearson's correlation coefficients. Bivariate and then multivariate logistic regression models assessed the predictors of cranial tremor in relatives. We used these models to assess the predictors of cranial tremor in relatives, using the presence versus absence of cra- 
Table 1. Demographic and clinical characteristics of enrollees

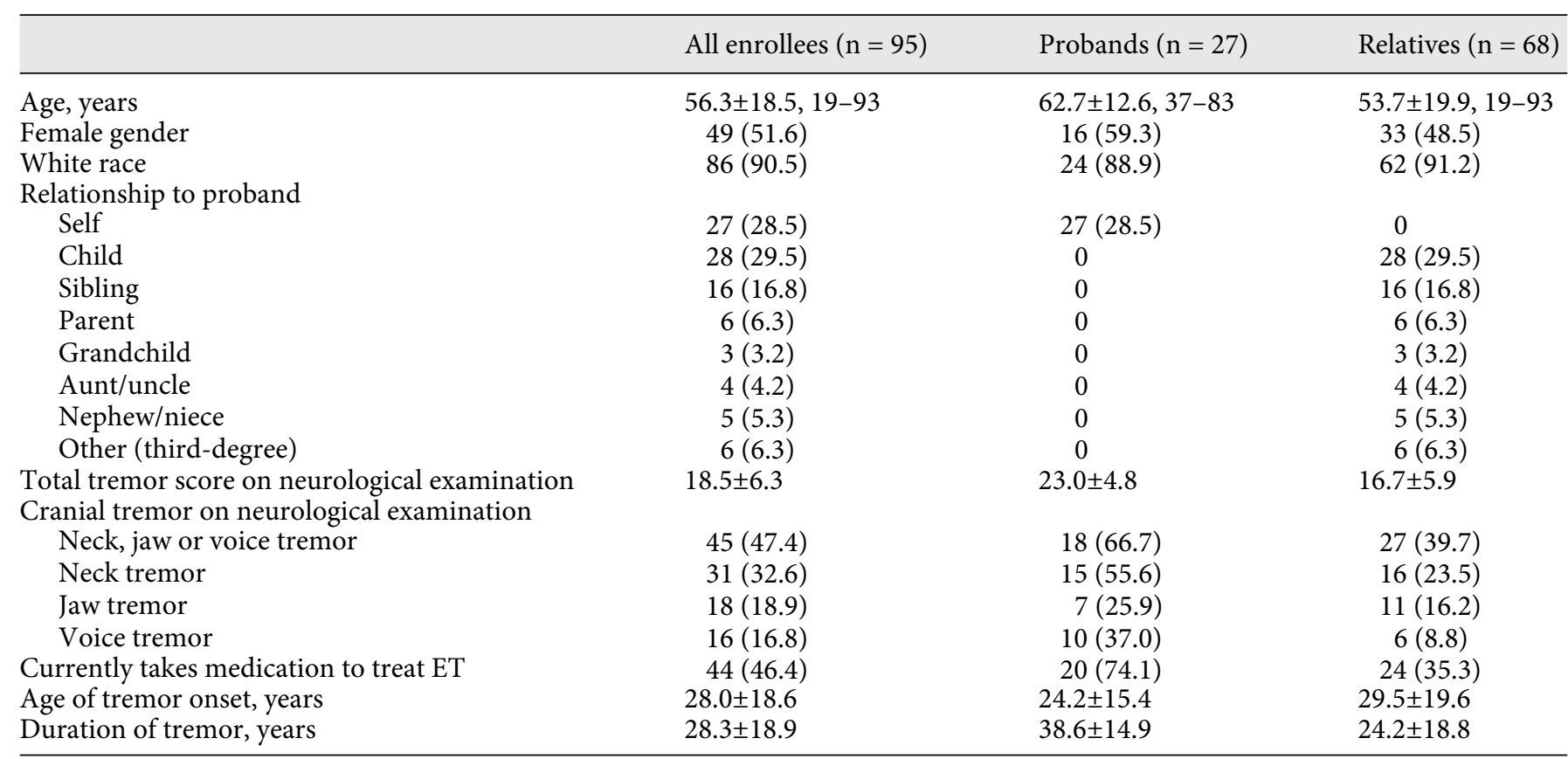

All values are means \pm standard deviation, range or number (\%), unless otherwise specified.

nial tremor in the proband as a primary predictor of interest. Other predictors that we considered included relative's age, total tremor score, tremor duration, and gender. Because of the non-independence of proband-relative pairs within each family, we used generalized estimating equations (GEEs) to compute odds ratios (ORs) and 95\% confidence intervals (CIs).

\section{Results}

\section{Prevalence and Correlates of Cranial Tremor}

The 68 relatives included 28 (41.2\%) children, 16 (23.5\%) siblings, and $6(8.8 \%)$ parents, with the remainder comprising other types of relatives (e.g. aunts/ uncles).

Forty-five subjects (18 probands and 27 relatives) had cranial tremor on neurological examination (table 1). Compared with the 50 subjects without cranial tremor, the 45 with cranial tremor had higher total tremor scores $(20.9 \pm 6.5$ vs. $16.3 \pm 5.3, t=3.72, \mathrm{p}<0.001)$, were older $(62.5 \pm 18.2$ vs. $50.7 \pm 17.1$ years, $\mathrm{t}=3.25, \mathrm{p}=0.003)$, and had tremor of longer duration ( $33.3 \pm 17.7$ vs. $23.8 \pm 18.9$ years, $\mathrm{t}=2.51, \mathrm{p}=0.01)$. Cranial tremor was more prevalent in women than men [26 (53.1\%) women vs. 19 $(41.3 \%)$ men, but not to a significant degree, $\chi^{2}=1.32, p=$ $0.25]$, and in particular, head tremor was more common in women [20 (40.8\%) vs. $11(23.9 \%), \chi^{2}=3.08, \mathrm{p}=0.079$ ] Total tremor score, age, and duration were collinear (all pairwise Pearson's $\mathrm{r}>0.51, \mathrm{p}<0.001$ ). The association between total tremor score and presence of cranial tremor is shown by family; in most families, the individuals with cranial tremor were those with the highest tremor scores (fig. 1). Similar relationships were seen when age and duration were plotted by presence of cranial tremor within families (data not shown).

\section{Familial Aggregation of Cranial Tremor}

Twenty-three (82.1\%) of 28 families included both individuals with and without cranial tremor, indicating a high degree of within-family heterogeneity. Three families (Nos. 20, 24, 28) were completely concordant for presence of cranial tremor (i.e. each family contained 2 affected family members both of whom had cranial tremor). Two families (Nos. 21 and 23) were concordant for absence of cranial tremor. Eighteen (66.7\%) of 27 probands versus $27(39.7 \%)$ of 68 relatives had cranial tremor $\left(\chi^{2}=5.63, p=0.018\right)$. With regard to presence versus absence of cranial tremor, $25(36.8 \%)$ relatives were concordant with their proband, 38 (55.9\%) relatives were discordant with their proband, and 5 relatives (family 23) did not have a proband with ET. 
Fig. 1. The association between tremor severity and presence of cranial tremor in 28 ET families. Total tremor score (y-axis) by family No. (x-axis and vertical lines). Subjects with cranial tremor are represented by closed boxes and subjects without cranial tremor are represented by open boxes. In most families, individuals with cranial tremor were those with the highest tremor scores. In 10 instances, $\geq 2$ individuals within a family had the same total tremor score and are represented by a single data point.

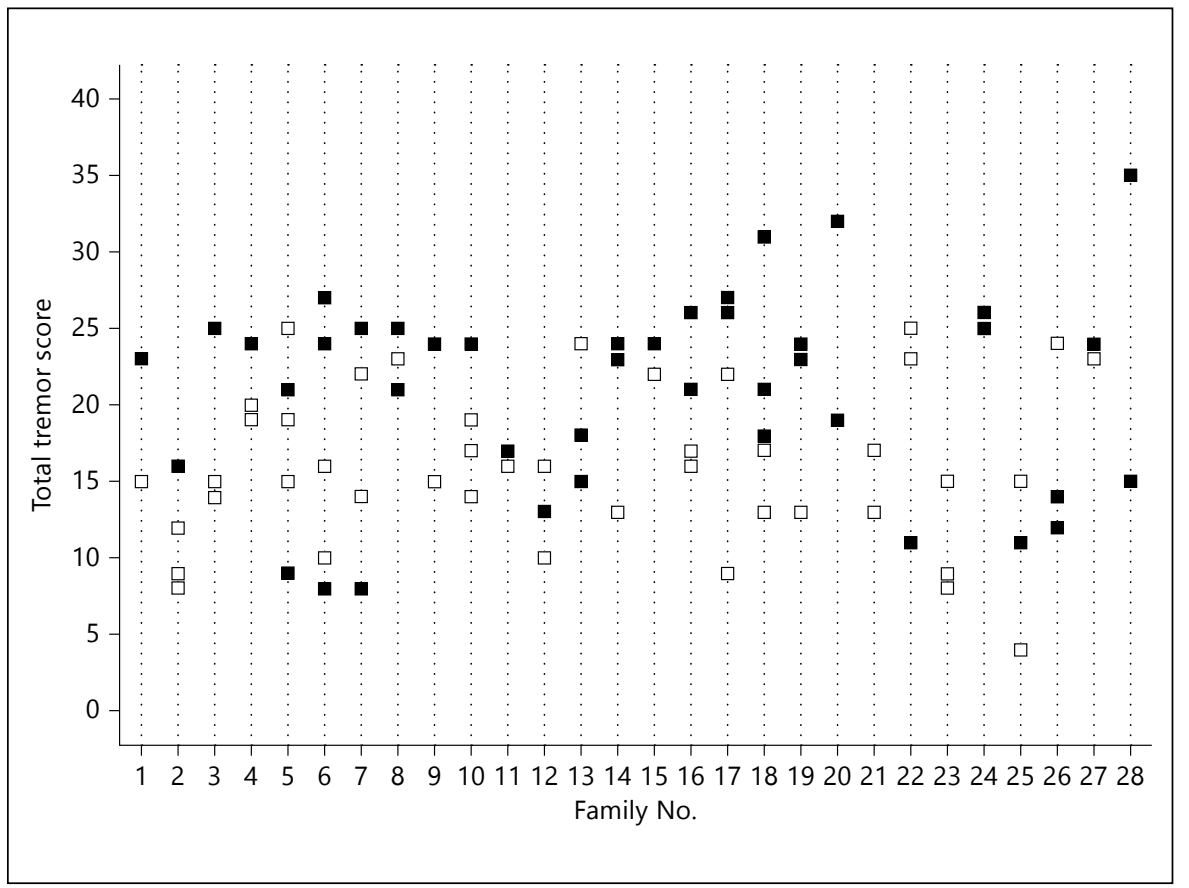

Predictors of Cranial Tremor in Relatives: Regression Modeling

Presence of cranial tremor in the relative (outcome variable) was associated with the relative's age $(\mathrm{OR}=1.03$, $95 \% \mathrm{CI}=1.003-1.06, \mathrm{p}=0.03)$ and tremor duration $(\mathrm{OR}=$ $1.03,95 \% \mathrm{CI}=1.00-1.06, \mathrm{p}=0.047)$. The strongest predictor of cranial tremor in the relative was the relative's total tremor score $(\mathrm{OR}=1.1,95 \% \mathrm{CI}=1.01-1.22, \mathrm{p}=0.035)$.

Presence of cranial tremor in relatives was not associated with presence of cranial tremor in the proband $(\mathrm{OR}=0.7,95 \% \mathrm{CI}=0.36-1.40, \mathrm{p}=0.32)$. This result was not substantially changed after adjusting for the relative's total tremor score $(\mathrm{OR}=0.8,95 \% \mathrm{CI}=0.37-1.58$, $\mathrm{p}=0.47)$, relative's tremor duration $(\mathrm{OR}=0.9,95 \% \mathrm{CI}=$ $0.45-1.88, \mathrm{p}=0.82)$, or relative's age $(\mathrm{OR}=0.6,95 \% \mathrm{CI}=$ $0.26-1.40, \mathrm{p}=0.24)$ in multivariate models. The relative's gender was not a confounder either. Total tremor score, age, and duration could not be included in the same model because they were collinear.

\section{Additional Analyses}

The large majority of individuals with cranial tremor had neck tremor. In a parallel set of analyses, we examined the predictors of neck tremor, and results were similar. Presence of neck tremor in relatives was not associated with presence of neck tremor in the proband $(\mathrm{OR}=0.6,95 \% \mathrm{CI}=0.23-1.63, \mathrm{p}=0.33)$. Adjusting for potential confounders (relative's total tremor score, relative's tremor duration, and relative's age) did not change the results. There was no confounding by gender.

\section{Discussion}

Head tremor is highly prevalent in ET, occurring in $30-50 \%$ of cases $[15,21]$. In addition to its reported clinical correlates [4-6], imaging studies have suggested that ET patients with head tremor may form a distinct subgroup, with reductions in cerebellar volume relative to those without head tremor $[22,23]$. The presence/absence of cranial tremors is also often used as a stratification point in genetic studies of ET [24, 25]. Yet, there are no readily available data on the concordance for such tremors in ET patients and their affected family members.

A large population-based family study in Sweden in the late 1950s included phenotype information on approximately 200 ET cases descended from a small number of ancestral families, and cranial tremors were reportedly present in some members of some families but not others, suggesting, as observed in our families, a high degree of within-family heterogeneity with respect to cranial tremor in ET, although the investigators did not consider the contributions of subject age, gender, duration, and other factors in their analyses [26]. Aside from that 
study, we are not aware of any prior published data on the concordance for cranial tremors in ET families.

ET patients whose relatives (e.g. parents) have/had head tremor often ask whether they, too, are likely to develop a similar head bobble. In the absence of published data, it has not been clear how to counsel these patients. The current data suggest that the development of head tremor is not strongly linked with family history, but rather, it seems to be more dependent on the extent of tremor severity and disease progression.

Eighteen (66.7\%) of 27 probands versus 27 (39.7\%) of 68 relatives had cranial tremor. Probands in this study may have self-selected based on the severity of their disorder, resulting in a high proportion with cranial tremor.

This study had limitations. Sample size was limited due to our restriction of the study to individuals who received in-person examination rather than including individuals with self-reported or proband-reported ET [27]. Despite the modest sample size, we were able to detect several important associations. Our restrictive inclusion criteria may also be viewed as a study strength, because they probably increased the validity of our diagnoses. Further strengths included the presence of multi-case families and a broad range of phenotypic features.

In summary, the familial aggregation of cranial tremor was low in ET. The major predictor of this disease feature was tremor severity rather than the presence of cranial tremor in another family member.

\section{Acknowledgement}

This research was supported by National Institutes of Health grant R01 NS073872 (E.D.L., L.N.C., and R.O.).

E.D.L. has received research support from the National Institutes of Health: NINDS R01 NS042859 (principal investigator; PI), NINDS R01 NS39422 (PI), NINDS T32 NS07153-24 (PI), NINDS R01 NS073872 (co-principal investigator; CoPI), NINDS R21 NS077094 (co-investigator; CoI), and NINDS R01 NS36630 (CoI), as well as the Parkinson's Disease Foundation (PI), the Arlene Bronstein Essential Tremor Research Fund (Columbia University), and the Claire O'Neil Essential Tremor Research Fund (Columbia University).

L.N.C. has received research support from National Institutes of Health grants R21NS050487 (PI), R01NS060113 (PI), R01NS0738072 (CoPI), P50AG008702 (CoI), P50 NS038370 (CoI), the Parkinson's Disease foundation (PI), and the Michael J. Fox foundation (CoI).

R.O. has received research support from the National Institutes of Health: NINDS R01 NS073872 (CoI), NINDS R01 NS053998 (CoI), NINDS R03 NS065346 (PI), NINDS RC2 NS070344 (PI), NHGRI P20 HG005535 (CoI), NINDS U01 NS077276 (PI), NINDS U01 NS077367 (PI), NINDS R01 NS078419 (PI), and NIA R01 AG041797 (CoI).

\section{Disclosure Statement}

The authors declare that there are no conflicts of interest and no competing financial interests.

\section{References}

1 Critchley M: Observations of essential (heredofamilial) tremor. Brain 1949;72:113139.

2 Benito-Leon J, Louis ED: Clinical update: diagnosis and treatment of essential tremor. Lancet 2007;369:1152-1154.

3 Louis ED, Rios E, Applegate LM, Hernandez NC, Andrews HF: Jaw tremor: prevalence and clinical correlates in three essential tremor case samples. Mov Disord 2006;21:18721878.

4 Louis ED, Rios E, Rao AK: Tandem gait performance in essential tremor: clinical correlates and association with midline tremors. Mov Disord 2010;25:1633-1638.

5 Parisi SL, Heroux ME, Culham EG, Norman KE: Functional mobility and postural control in essential tremor. Arch Phys Med Rehabil 2006;87:1357-1364.

6 Hoskovcova M, Ulmanova O, Sprdlik O, Sieger T, Novakova J, Jech R, Ruzicka E: Disorders of balance and gait in essential tremor are associated with midline tremor and age. Cerebellum 2013;12:27-34.
7 Putzke JD, Uitti RJ, Obwegeser AA, Wszolek ZK, Wharen RE: Bilateral thalamic deep brain stimulation: midline tremor control. J Neurol Neurosurg Psychiatry 2005;76:684690.

8 Song IU, Kim JS, Lee SB, Ryu SY, An JY, Kim HT, Kim YI, Lee KS: Effects of zonisamide on isolated head tremor. Eur J Neurol 2008; 15: 1212-1215.

9 Zesiewicz TA, Elble R, Louis ED, Hauser RA, Sullivan KL, Dewey RB Jr, Ondo WG, Gronseth GS, Weiner WJ: Practice parameter: therapies for essential tremor: report of the Quality Standards Subcommittee of the American Academy of Neurology. Neurology 2005;64: 2008-2020.

10 Louis ED, Ottman R: How familial is familial tremor? The genetic epidemiology of essential tremor. Neurology 1996;46:1200-1205.

11 Tan EK, Schapira AH: Hunting for genes in essential tremor. Eur J Neurol 2008;15:889890.
12 Louis ED, Hernandez N, Ionita-Laza I, Ottman R, Clark LN: Does rate of progression run in essential tremor families? Slower vs. faster progressors. Parkinsonism Relat Disord 2013;19:363-366.

13 Louis ED, Hernandez N, Rabinowitz D, Ottman R, Clark LN: Predicting age of onset in familial essential tremor: how much does age of onset run in families? Neuroepidemiology 2013;40:269-273.

14 Louis ED, Hernandez N, Alcalay RN, Tirri DJ, Ottman R, Clark LN: Prevalence and features of unreported dystonia in a family study of 'pure' essential tremor. Parkinsonism Relat Disord 2013;19:359-362.

15 Louis ED, Ford B, Frucht S: Factors associated with increased risk of head tremor in essential tremor: a community-based study in northern Manhattan. Mov Disord 2003;18:432-436.

16 Louis ED, Dogu O: Does age of onset in essential tremor have a bimodal distribution? Data from a tertiary referral setting and a population-based study. Neuroepidemiology 2007;29:208-212. 
17 Louis ED, Ottman R, Ford B, Pullman S, Martinez M, Fahn S, Hauser WA: The Washington Heights-Inwood genetic study of essential tremor: methodologic issues in essentialtremor research. Neuroepidemiology 1997; 16:124-133.

18 Louis ED, Schonberger RB, Parides M, Ford B, Barnes LF: Test-retest reliability of patient information on age of onset in essential tremor. Mov Disord 2000;15:738-741.

19 Louis ED, Jiang W, Pellegrino KM, Rios E, Factor-Litvak P, Henchcliffe C, Zheng W: Elevated blood harmane (1-methyl-9h-pyrido[3, 4-b]indole) concentrations in essential tremor. Neurotoxicology 2008;29:294300.

20 Louis ED, Ford B, Bismuth B: Reliability between two observers using a protocol for diagnosing essential tremor. Mov Disord 1998; 13:287-293.
21 Hardesty DE, Maraganore DM, Matsumoto JY, Louis ED: Increased risk of head tremor in women with essential tremor: longitudinal data from the Rochester Epidemiology Project. Mov Disord 2004; 19:529-533.

22 Cerasa A, Messina D, Nicoletti G, Novellino F, Lanza P, Condino F, Arabia G, Salsone M, Quattrone A: Cerebellar atrophy in essential tremor using an automated segmentation method. AJNR 2009;30:1240-1243.

23 Quattrone A, Cerasa A, Messina D, Nicoletti G, Hagberg GE, Lemieux L, Novellino F, Lanza P, Arabia G, Salsone M: Essential head tremor is associated with cerebellar vermis atrophy: a volumetric and voxel-based morphometry MR imaging study. AJNR 2008;29: 1692-1697.
24 Garcia-Martin E, Martinez C, Alonso-Navarro H, Benito-Leon J, Puertas I, Rubio L, Lopez-Alburquerque T, Agundez JA, JimenezJimenez FJ: Dopamine receptor D3 (DRD3) genotype and allelic variants and risk for essential tremor. Mov Disord 2009;24:19101915.

25 Alonso-Navarro H, Martinez C, Garcia-Martin E, Benito-Leon J, Garcia-Ferrer I, VazquezTorres P, Puertas I, Lopez-Alburquerque T, Agundez JA, Jimenez-Jimenez FJ: CYP2C19 polymorphism and risk for essential tremor. Eur Neurol 2006;56:119-123.

26 Larsson T, Sjogren T: Essential tremor: a clinical and genetic population study. Acta Psychiatr Scand Suppl 1960;36:1-176.

27 Louis ED, Ford B, Pullman SL: Prevalence of asymptomatic tremor in relatives of patients with essential tremor. Arch Neurol 1997;54: 197-200. 